\title{
PHOTOEXCITED TRANSIENTS IN DISORDERED SEMICONDUCTORS: KINEMATICALLY INDUCED ELECTRON-HOLE CORRELATION *
}

\author{
A. KALVOVÁ \\ Institute of Physics, Academy of Sciences of the Czech Republic, \\ $\mathrm{Na}$ Slovance 2, \\ CZ-180 40 Praha 8, Czech Republic \\ E-mail: kalvova@fzu.cz \\ B. VELICKÝ \\ Institute of Physics, Academy of Sciences of the Czech Republic, \\ $\mathrm{Na}$ Slovance 2, \\ CZ-180 40 Praha 8 \\ and \\ Faculty of Mathematics and Physics, Charles University, \\ Ke Karlovu 5, \\ CZ-121 16 Praha 2, Czech Republic \\ E-mail:velicky@karlov.mff.cuni.cz
}

\begin{abstract}
In mixed semiconductor crystals, the alloy disorder in the valence and conduction bands is statistically correlated. This leads to kinematical correlations in the motion of electrons and holes analogous to exciton effects. This mechanism known to affect the linear optical response is presently shown to act also in the non-linear regime induced by strong short light pulses. A direct numerical solution of the Kadanoff-Baym equations for non-equilibrium Green functions (with a self-consistent and conserving single-site approximation for the self-energy) is used to study correlation in the electron and hole photoexcited populations, and also an additional transient light induced band hybridization which modifies the full optical vertex.
\end{abstract}

\section{Introduction}

In the linear optical response of electrons in disordered semiconductors, the statistical correlation between the disorder in the valence and conduction bands leads to a kinematical correlation between the electrons and

*based on a poster presented at the conference "progress in nonequilibrium greens functions, dresden, germany, 19.-22. august 2002" 
the holes. They behave as if a peculiar $e-h$ interaction acted within the photoexcited electron - hole pair. This may be described, in analogy to the true excitons, by an optical vertex, or, equivalently, by the so-called Elliott factor ${ }^{1}$ entering the Golden Rule matrix element. Sometimes, near the absorption edge, this kinematic effect combines with a true screened Coulomb interaction between both particles. At higher excitation energies, the dynamic effect is negligible and only the kinematic coupling remains. These phenomena were studied in detail long time ago, in connection with the problem of the optical absorption in disordered (amorphous) semiconductors. We refer the reader to two chapters, 2 and 8, of the book ${ }^{2}$. We have to distinguish between the case of smooth random fields, due either to space charges, or to internal strains, typical for amorphous semiconductors, and the case of short range random fluctuations, related to the chemical (substitutional) disorder on an atomic scale in semiconductor alloys. The first case can be treated using the effective mass picture combined with a quasiclassical approximation. We will be interested in the other case of semiconductor alloys, which requires a fully quantum treatment, based typically on a single site approximation of the CPA character. This is reviewed in Ref. ${ }^{3}$. Linear optical transitions are reviewed in Ref. ${ }^{4}$, directly related papers are ${ }^{5},{ }^{6},{ }^{7}$

The kinematic correlation mechanism, as will be demonstrated presently, acts also in the non-linear regime induced by strong short optical pulses. The new features as compared with the linear regime concern the correlation in the electron and hole photoexcited populations, and also an additional transient light induced band hybridization which modifies the full optical vertex.

For the early time range when the disorder is still the dominant source of scattering, the problem is conveniently treated by a direct solution of the Kadanoff-Baym (differential Dyson) equations for non-equilibrium Green functions. We concentrate to substitutional alloys modelled by a two band Hamiltonian reasonably close to a realistic description and employ a selfconsistent and conserving single-site approximation for the self-energy. The disorder strength is chosen such that the picture of complex bands can be used and the effects of localization and impurity bands are not important. At the same time, the disorder dominates the optical transient and is predicted to have pronounced consequences. The present paper is an extension of our previous work, and much of the physical discussion can be found in paper $^{8}$.

We first specify our two band model in Sec. 2 and indicate the link between the disorder induced optical vertex in linear response and in non- 
linear transients in Sec 3. Then the formulation based on the one-electron NGF is sketched in Sec. 4. The last section 5 is devoted to the physical discussion of the kinematical correlation based on numerical results.

\section{Model semiconductor alloy}

Following ${ }^{8}$, we consider a completely random binary alloy $\mathrm{A}_{c^{A}} \mathrm{~B}_{c^{B}}$ with $c^{A}+c^{B}=1$. The semiconductor has a two-band electron structure with the gap between two isotropic parabolic band edges at the center of the Brillouin zone ("standard band structure"). All many-body interactions are ignored. The disorder potential acts in each band separately, not mixing states of both bands ("independent bands"). The effect of the optical pulse is restricted to a non-random interband dipole coupling treated in the rotating wave approximation (RWA). The initial condition is a fully occupied valence band and a completely empty conduction band before the arrival of light.

The notion of independent bands is formalized by introducing the band projectors which are non-random and diagonal in both the Bloch and the Wannier (site) basis:

$$
P_{b}=\sum_{\mathrm{BZ}}|b \mathrm{k}\rangle\left\langle b \mathrm{k}\left|=\sum_{\text {lattice }}\right| b i\right\rangle\langle b i|, \quad b=c, v .
$$

The full one-electron Hamiltonian for one configuration of our alloy has the following structure:

$$
\mathcal{H}=W_{v}+W_{c}+\mathcal{V}_{c}+\mathcal{V}_{v}+U(t) \equiv \mathcal{H}_{c}+\mathcal{H}_{v}+U(t) \equiv \mathcal{H}_{\text {DARK }}+U(t)
$$

The non-random configuration independent or averaged quantities are denoted by italics, while the configuration dependent ones by rounded (" Ical ") characters. The dark Hamiltonian $\mathcal{H}_{\text {DARK }}$ is band diagonal, as both the Bloch Hamiltonians $W_{b}$ and the random potentials $\mathcal{V}_{b}$ are assumed to obey the rule $\mathcal{O}_{b}=P_{b} \mathcal{O}_{b} P_{b}$ with $b=c, v$. The interaction with the pulse is taken as purely interband, that is off-diagonal; with the linear polarization in the $x$ direction, the basic frequency $\Omega$, and in the Rotating Wave Approximation, it reads

$$
\begin{aligned}
U(t) & =U_{c v}(t)+U_{v c}(t) \\
& =-Q \cdot \Phi(t)\left\{\sum_{k}|c \mathrm{k}\rangle \mathrm{e}^{-i \Omega t}\left\langle v \mathrm{k}\left|+\sum_{k}\right| v \mathrm{k}\right\rangle \mathrm{e}^{i \Omega t}\langle c \mathrm{k}|\right\} \\
& \equiv-Q \cdot \Phi(t)\left\{\Pi_{c v} \mathrm{e}^{-i \Omega t}+\Pi_{v c} \mathrm{e}^{+i \Omega t}\right\}
\end{aligned}
$$

where $Q=e x_{c v} \mathrm{E}_{\mathrm{M}}$ is the strength of the pulse, $e x_{c v}$ a real constant electric dipole matrix element and $\mathrm{E}_{\mathrm{M}}$ the peak electric field of the pulse; $\epsilon_{x}(t)=$ $\mathrm{E}_{\mathrm{M}} \Phi(t) \geq 0$ is the pulse envelope. 


\section{Optical vertex in linear and non-linear response}

In general, the macroscopic optical response is given by the electric polarization $\boldsymbol{P}$ induced by the electric field $\boldsymbol{E}$ of incident light. In our isotropic case, we need

$$
P_{x}(t)=\left(N \Upsilon_{0} \epsilon_{0}\right)^{-1} \operatorname{Tr}\langle e \hat{x} \cdot \varrho(t)\rangle
$$

with $\hat{x}=x_{c v}\left\{\Pi_{c v}+\Pi_{v c}\right\}$, and $\varrho$ the one-electron density matrix for a random atomic configuration. $\langle\cdots\rangle$ indicates averaging over these random configurations. $N$ is the number of primitive cells, $\Upsilon_{0}$ the cell volume. The electric field enters through the time dependence of $\varrho$. The initial condition is $\varrho\left(t_{0}\right)=P_{v}$ for $t_{0}$ in a distant past. Clearly, $P_{x}\left(t_{0}\right)=0$.

Some understanding of (4) can be gained in the linear case. Then, $\boldsymbol{P}$ and $\boldsymbol{E}$ are linked by the dielectric function $\varepsilon$ defined by a symbolic equation $\boldsymbol{P}=\epsilon_{0}(\varepsilon-1) \cdot \boldsymbol{E}$. The absorptive part of its Fourier transform, the complex permittivity, for our model can be rewritten ${ }^{4}$ to reflect the simultaneous motion of the photoexcited $e-h$ pair:

$$
\begin{aligned}
\varepsilon_{2}(\omega) & =2\left(N \Upsilon_{0} \epsilon_{0}\right)^{-1} \int d \eta \operatorname{Tr}\left\langle\delta\left(\eta-\mathcal{H}_{v}\right) \Pi_{v c} \delta\left(\eta+\hbar \omega-\mathcal{H}_{c}\right) \Pi_{c v}\right\rangle \\
& =2\left(N \Upsilon_{0} \epsilon_{0}\right)^{-1} \int d \eta \sum_{\mathrm{k}} A_{v}(\mathrm{k}, \eta)\left[1+\Gamma_{v c}(\mathrm{k}, \eta, \eta+\hbar \omega)\right] A_{c}(\mathrm{k}, \eta+\hbar \omega)
\end{aligned}
$$

Before averaging, the electron and the hole move independently in their respective bands. Configuration average of the product of two $\delta$-functions would factorize only if the randomness in both bands were uncorrelated. In a chemical environment common for both particles, a disorder induced optical vertex $\Gamma_{v c}$ modifies the product of the averaged $\delta$-functions $A_{b}(E)=$ $\left\langle\delta\left(E-\mathcal{H}_{b}\right)\right\rangle$. Quantities $A_{b}(E)$ have the meaning of spectral densities in the individual bands. They describe the band broadening and shift, in other words the polaron-like effects of the disorder. In Eq. (5), they give rise to non-direct (non-vertical) transitions. The vertex $\Gamma_{v c}$ plays the role of a generalized Elliott factor, as it describes the joint behavior of the electron and the hole. It depends on the degree of stochastic correlation between both band Hamiltonians ${ }^{7},{ }^{6},{ }^{4}$.

There are several ways of transforming Eq. (4) in the non-linear case to equations resembling (5). There are important qualitative differences, however. These equations have to be written in the time domain, with the spectral densities replaced by configuration averaged propagators. The propagators and the vertex kernels are dressed by the light ... the nonlinear effect. As a consequence, the propagator has also band off-diagonal blocks, which describe the light induced hybridization of both bands and cannot be interpreted as a propagation of a well defined $e-h$ pair. While 
these results are physically revealing, they offer no easy way for an actual evaluation. For that we turn to the NGF formulation.

\section{Equations for NGF including kinematical correlations}

We work in the standard LW matrix form of NGF. For example:

$$
\mathbf{G}=\left\|\begin{array}{cc}
G^{R} & G^{<} \\
0 & G^{A}
\end{array}\right\| ; \quad \boldsymbol{\Sigma}=\left\|\begin{array}{cc}
\Sigma^{R} & \Sigma^{<} \\
0 & \Sigma^{A}
\end{array}\right\| ; \quad \mathcal{H}=\left\|\begin{array}{cc}
\mathcal{H} & 0 \\
0 & \mathcal{H}
\end{array}\right\|
$$

The Hamiltonians are split into their averaged (mean field, virtual crystal) and fluctuating parts:

$$
\left.\begin{array}{c}
\mathcal{H}=\langle\mathcal{H}\rangle+\mathcal{D} \\
\mathcal{H}_{\text {DARK }}=\left\langle\mathcal{H}_{\text {DARK }}\right\rangle+\mathcal{D}
\end{array}\right\} \mathcal{D}=\mathcal{D}_{v}+\mathcal{D}_{c} \equiv\left(\mathcal{V}_{v}-\left\langle\mathcal{V}_{v}\right\rangle\right)+\left(\mathcal{V}_{c}-\left\langle\mathcal{V}_{c}\right\rangle\right)
$$

The Dyson equation for $\mathbf{G}$ is rewritten in terms of $\mathbf{G}_{\mathrm{DARK}}$ :

$$
\begin{aligned}
\mathbf{G}^{-1} & =i \hbar \partial_{t} \mathbf{1}-\left\langle\boldsymbol{\mathcal { H }}_{\mathrm{DARK}}\right\rangle-\mathbf{U}-\boldsymbol{\Sigma} \\
& =\mathbf{G}_{\mathrm{DARK}}^{-1}-\mathbf{U}-(\underbrace{\boldsymbol{\Sigma}-\boldsymbol{\Sigma}_{\text {DARK }}}_{\boldsymbol{\Sigma}_{\mathrm{IND}}})
\end{aligned}
$$

The dark quantities are known beforehand and we solve numerically the Dyson equation in the second form together with the self-consistent Born approximation for the light induced part of the self-energy,

$$
\begin{aligned}
\boldsymbol{\Sigma}_{\mathrm{IND}} & =\left\langle\mathcal{D}\left(\mathbf{G}-\mathbf{G}_{\mathrm{DARK}}\right) \mathcal{D}\right\rangle= \\
\left\langle\left[\mathcal{D}_{v}+\mathcal{D}_{c}\right]\left(\mathbf{G}-\mathbf{G}_{\mathrm{DARK}}\right)\left[\mathcal{D}_{v}+\mathcal{D}_{c}\right]\right\rangle & =\boldsymbol{\Sigma}_{\mathrm{IND} v v}+\boldsymbol{\Sigma}_{\mathrm{IND} v c}+\boldsymbol{\Sigma}_{\mathrm{IND} c v}+\boldsymbol{\Sigma}_{\mathrm{IND} c c}
\end{aligned}
$$

The off-diagonal parts of $\boldsymbol{\Sigma}_{\text {IND }}$ are the explicit source of the disorder induced correlation. It spreads, by self-consistence, also to the diagonal parts. We consider the case of a "diagonal disorder", when only the quasiatomic levels in the Wannier representation fluctuate. For a binary alloy, let the levels be $E_{c n}=E_{c}^{A}, E_{c}^{B} ; E_{v n}=E_{v}^{A}, E_{v}^{B}$ at random. The measure of the intraband disorder, and of the cross-correlation, according to (9), is

$$
\begin{aligned}
\left\langle\left(E_{v n}-\left\langle E_{v n}\right\rangle\right)\right\rangle^{2} & =c^{A} c^{B}\left(E_{v}^{A}-E_{v}^{B}\right)^{2} \equiv \lambda_{v}^{2} \\
\left\langle\left(E_{c n}-\left\langle E_{c n}\right\rangle\right)\right\rangle^{2} & =c^{A} c^{B}\left(E_{c}^{A}-E_{c}^{B}\right)^{2} \equiv \lambda_{c}^{2} \\
\left\langle\left(E_{v n}-\left\langle E_{v n}\right\rangle\right)\left(E_{c n}-\left\langle E_{c n}\right\rangle\right)\right\rangle & =c^{A} c^{B}\left(E_{v}^{A}-E_{v}^{B}\right)\left(E_{c}^{A}-E_{c}^{B}\right) \equiv \lambda_{v} \lambda_{c} \kappa
\end{aligned}
$$

Depending on the sign interplay of the level differences, the correlation parameter $\kappa$ is \pm 1 . We will compare three cases:

\begin{tabular}{|c|c|c|}
\hline$\kappa=-1$ & 0 & 1 \\
anti-correlated & non-correlated & correlated \\
\hline
\end{tabular}



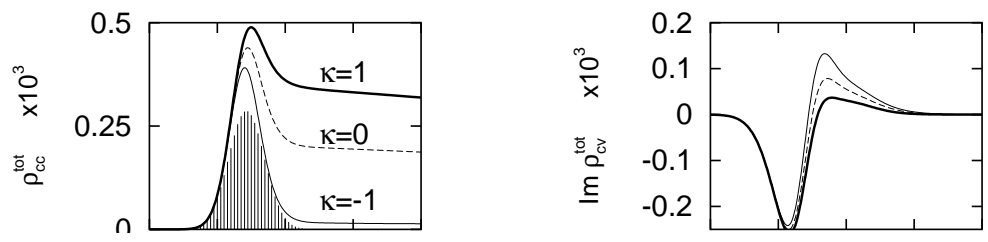

Figure 1. The total one-electron density matrix (12) as a function of time. The diagonals are real, the off-diagonals are complex conjugate, and we show the real and the imaginary parts separately. Three different cases of the " $e-h$ " correlation have a line code defined in the $\rho_{c c}^{\mathrm{TOT}}$ panel. The hatched profile: pulse intensity shape, arbitrary scale.

As will be seen, this statistical correlation can be reformulated in terms of "effective $e-h$ interaction" ${ }^{2}$ also in the non-linear case.

\section{Correlation dependent transient photoexcitation}

To demonstrate the importance of the $e-h$ disorder induced correlation, we present here a few numerical results obtained by solving the Eqs. (8) and (9) using the method described in ref. ${ }^{8}$. Primarily, we have calculated the quantities ${ }^{\kappa} G_{a b}^{R}\left(k ; t, t^{\prime}\right),{ }^{\kappa} G_{a b}^{<}\left(k ; t, t^{\prime}\right),{ }^{\kappa} \sum_{\mathrm{IND}, a b}^{R}\left(t, t^{\prime}\right),{ }^{\kappa} \sum_{\mathrm{IND}, a b}^{<}\left(t, t^{\prime}\right)$ for $a, b=c, v$ and $\kappa=0, \pm 1$. The calculations were performed for these parameters: $E_{G}=1.5 \mathrm{eV}, \hbar \Omega=2 . \mathrm{eV}, m_{v}^{*}=.6, m_{c}^{*}=.4$; disorder: $c$ and $v$ - band lifetimes $\tau_{c}=160 \mathrm{fs}, \tau_{v}=240 \mathrm{fs}, c^{A}=0.05$; pulse: Gaussian, duration $\tau_{m}=120 \mathrm{fs}$, Rabi phase $3.3 Q \tau_{m} / \hbar=1.93 \pi$. We cannot analyze here the results for the Green's functions and for the self-energies. Instead, we limit ourselves to presenting the behavior of observable quantities. The results are plotted in the Galickii picture suppresing the $\Omega$-oscillations.

The averaged one-electron density matrix is given by

$$
{ }^{\kappa} \rho(t)=-\mathrm{i} \hbar^{\kappa} G^{<}(t, t)
$$

It is $k$-, but not band-, diagonal. To obtain a summary information, we 
trace over the $k$-vectors:

$$
{ }^{\kappa} \rho_{a b}^{\mathrm{TOT}}(t)=N^{-1} \sum_{k \in \mathrm{BZ}}{ }^{\kappa} \rho_{a b}(\mathrm{k}, t), \quad a, b=c, v
$$

The time evolution of ${ }^{\kappa} \rho^{\text {Tот }}$ is in Fig. 1 . The diagonal elements have the interpretation of the total photoexcited populations, namely $n_{e}=\rho_{c c}^{\text {Toт }}$, $n_{h}=1-\rho_{v v}^{\text {TOT }}$. The effect of correlation is profound. Physically more relevant are the off-diagonal elements linked with the electric polarization by $M_{x}(t)=e x_{c v} \cdot 2 \operatorname{Re}\left[\rho_{c v}^{\mathrm{ToT}} \mathrm{e}^{-i \Omega t}\right]$ (the dipole moment of one cell) and $P_{x}=\Upsilon_{0}^{-1} M_{x}$. The populations and the polarization have in common that they are correlation insensitive early after the pulse arrival, while the differences are marked at late times, in a qualitative agreement with the notion of final state $e-h$ interactions. This is explained by linking the correlation effects with the incoherent back-scattering.

The tendency is different, however. The diagonal elements increase with a stronger correlation, while the off-diagonal ones decrease. This may be partly understood by considering the energy balance. The "Joule heat" rate (actually, a non-dissipative energy $\operatorname{transfer}^{8}$ ) is

$$
w=\boldsymbol{E} \cdot \boldsymbol{J}=\boldsymbol{E} \cdot \frac{\partial}{\partial t} \boldsymbol{P}
$$

As seen in Fig. 2, the gain becomes negative at the trailing side of the
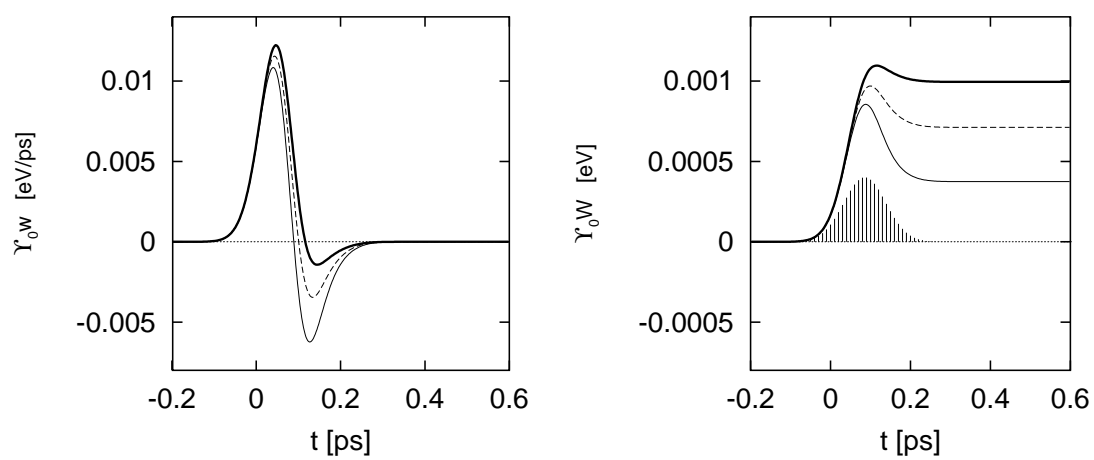

Figure 2. The absorbed power (left panel) and the integral energy gain (right panel) obtained as "Joule heat" from Eq. (14 ). Line code as in Fig. 1.: thick line $\cdots \kappa=1$, thin line $\cdots \kappa=-1$, dashed line $\cdots \kappa=0$. Hatched profile: pulse amplitude, arbitrary scale 
pulse, more for $\kappa=-1$ and less for $\kappa=1$. By

$$
W(t)=\int_{-\infty}^{t} \mathrm{~d} t^{\prime} w\left(t^{\prime}\right)
$$

this is converted into a reverted order of magnitude of the integral energy transfer. The tentative interpretation of this behavior is that positive/negative correlation enhances/reduces the incoherent backscattering, and, hence, the disorder effect on the optical response.

\section{Conclusion}

(1) The stochastic correlation in motion of the photoexcited electron -hole pairs is predicted to have a strong effect on the absorption of light from a pulse

(2) The theory developed presently extends the previous work for the linear response ${ }^{5},{ }^{6}$ to non-linear transients ${ }^{8}$

(3) Main effect of the $e-h$ kinematic correlation is to modify the "long" time asymptotics corresponding to the final state interaction in the linear theory and to the incoherent backscattering in general

(4) correlated $e-h$ motion enhances the disorder effect ("attraction") anticorrelated $e-h$ motion reduces the disorder effect ("repulsion")

\section{Acknowledgments}

This work was supported by the Grant Agency of the Czech republic under the project number 202/00/0643.

\section{References}

1. R. J. Elliott, Phys. Rev. 108, 1384 (1957)

2. B. Seraphin, Editor, Optical properties of solids: New developments, North Holland/ American Elsevier, Amsterdam 1976

3. AB Chen and A. Sher, Semiconductor Alloys, Plenum, New York 1995

4. B. Velický and A. Pieczonková, Physica scripta T19, 558 (1987)

5. S. Abe and Y. Toyozawa, J. Phys. Soc. Japan 50, 2185 (1981)

6. K. C. Hass, Topics in the Electronic Theory of Disordered Semiconductors, Thesis, Ch. VIII., Harvard University, Cambridge 1984

7. N. F. Schwabe and R. J. Elliott, Phys. Rev. B 53, 5301 (1996)

N. F. Schwabe and R. J. Elliott, Phys. Rev. B 53, 5318 (1996)

8. A. Kalvová and B. Velický, Phys. Rev B 65155329 (2002) 
Index

Disordered semiconductors, 1

Elliott factor effective, 4

non-linear response, 4

optical transients, 6

optical vertex, 4

Electron-hole correlation

disorder induced, 2, 4

Joule heat, 8

optical transients, 7 